\title{
ỨNG DỤNG BAN ĐẦU HÓA XOÁY MÔ PHỎNG VÀ NGHIẾN CÚUU CẤU TRÚC BÃO MUJIGAE (2015) KHI GẦN BỜ VÀ ĐỔ Bộ
}

\author{
Nguyễn Bình Phong ${ }^{1,2}$, Nguyễn Tiến Mạnh ${ }^{1}$, Nguyễn Xuân Anh ${ }^{1}$ \\ Phạm Lê Khương ${ }^{1}$, Nguyễn Đức Nam ${ }^{1}$, Phạm Xuân Thành ${ }^{1}$, Nguyễn Văn Hiệp ${ }^{1}$
}

Tóm tắt: Bài báo này này úng dụng so đồ ban đầu hóa xoáy động lực với mô hình WRF (Weather Research Forecasting) nghiên cưu ảnh huởng của địa hình và gió mùa đông bắc tới cấu trúc bão Mujigae (2015). Kết quả cho thấy ban đầu hóa xoáy đã cải thiện đáng kể chất lượng điều kiện ban đầu với kích thước mắt bão và vùng mây trong bão tại thời điểm ban đầu phù hợp với quan trắc. Điều kiện ban đầu được cải thiện giúp mô hình mô phỏng quỹ đạo phù hợp với quan trắc hơn. Phân tích sản phẩm mô hình chạy với ban đầu hóa xoáy cho thấy cấu trúc hoàn luu bão Mujigae có tính phi đối xứng mạnh với mây và gió mạnh tập trung phía đông và phía bắc của tâm bão. Điểm đặc bất thuờng trong cấu trúc trường gió khi bão Mujigae đổ bộ là phần trên đất liền phía bắc tâm bão noi chịu ma sát mạn của đất liền lại có gió mạnh hơn phần trên biển nơi có ảnh hưởng ma sát yếu. Sự bất thường này có thể do gió phần phía bắc conn bão được tăng cuờng bởi gió gradient mạnh khi có sụ hoạt động của áp cao lạnh lục địa.

Từ khóa: Ban đầu hóa xoáy, Bão, WRF.

Ban Biên tập nhận bài: 12/11/2019 Ngày phản biện xong: 28/12/2019 Ngày đăng bài: 25/01/2020

\section{Giới thiệu}

Bão là một trong những hiện tượng thời tiết nguy hiểm, không chỉ gây ra những thiệt hại to lớn về kinh tế - xã hội mà còn đe dọa tính mạng của con người. Theo số liệu thống kê nhiều năm thì trung bình hàng năm có khoảng 5 - 6 cơn bão và 2 - 3 áp thấp nhiệt đới (ATNĐ) ảnh hưởng đến Việt Nam (http://www.kttvqg.gov.vn). Trong những năm gần đây, cường độ và quỹ đạo của bão ngày càng có diễn biến phức tạp, gây khó khăn cho công tác dự báo, đồng thời cũng gây ra thiệt hại khó lường. Vì vậy, nhằm giảm thiểu hậu quả do bão gây ra, công tác dự báo bão đang được quan tâm tại nhiều quốc gia trên thế giới, trong đó có Việt Nam.

Như chúng ta đã biết, sự hình thành và phát triển của bão thường diễn ra trên biển, nơi mà

${ }^{1}$ Viện Vật lý địa cầu, Viện Hàn lâm Khoa học và Công nghệ Việt Nam

${ }^{2}$ Trường Đại học Tài nguyên và Môi trường Hà Nội

Email:hiepwork@gmail.com nguồn số liệu quan trắc vô cùng thưa thớt. Hơn nữa, điều kiện ban đầu của các mô hình khu vực được nội suy từ phân tích toàn cầu có độ phân giải tương đối thô, điều đó có thể dẫn đến sự khác biệt lớn về cường độ cũng như là đặc điểm vật lý giữa xoáy ban đầu trong mô hình khu vực và mô hình toàn cầu. Ngoài ra, xoáy ban đầu từ phân tích toàn cầu thường có cường độ quá yếu hoặc quá mạnh so với thực tế, đây là một trong những nguyên nhân dẫn đến những sai số trong quá trình khởi tạo Kurihara và cộng sự (1993) [10].

Ban đầu hóa xoáy là kĩ thuật xây dựng một xoáy giả có cấu trúc và cường độ gần với xoáy bão thực, có vị trí trùng với vị trí xoáy bão quan trắc để thay thế xoáy có độ phân giải thấp từ phân tích toàn cầu Mathur (1991) [13], Kurihara và cộng sự (1993) [11] nhằm cải thiện điều kiện ban đầu cho các mô hình số. Mathur (1991) [13] sử dụng hàm phân tích thực nghiệm cho cấu trúc gió từ bề mặt tới các mực trên cao cho quá trình ban đầu hóa, kết quả cho thấy sự cải thiện đáng kể trong điều kiện ban đầu của bão. 
Ngoài ra, quá trình ban đầu hóa xoáy trong mô hình số cũng đã góp phần cải thiện được mô phỏng và dự báo cường độ, quỹ đạo bão. Kwon và Cheong (2010) [12] đã đưa ra phương pháp ban đầu hóa xoáy với xoáy giả ba chiều lý tưởng từ dữ liệu thực nghiệm và dữ liệu phân tích. Nghiên cứu đã chỉ ra rằng ban đầu hóa xoáy đã cải thiện được đáng kể dự báo cường độ lẫn quỹ đạo bão so với trường hợp không có ban đầu hóa. Chou và cộng sự (2010) [9], Pu và cộng sự (2002) [16] đồng hóa dữ liệu giáng thủy bề mặt TRMM cho siêu bão Paka (1997) bằng mô hình MM5. Kết quả nghiên cứu cho thấy, đồng hóa dữ liệu góp phần cải thiện cường độ, cấu trúc động học và lượng mưa trong bão. Đồng thời cường độ và quỹ đạo dự báo cũng được cải thiện đáng kể. Wu và Chou (2008) [8] tiến hành ban đầu hóa xoáy bằng cách sử dụng kĩ thuật đồng hóa dữ liệu thám sát gió (dropwindsonde) cũng cho thấy những cải thiện về dự báo cường độ và quỹ đạo bão.

Tại Việt Nam, một số nghiên cứu về ban đầu hóa xoáy đã được thực hiện nhằm cải thiện chất lượng dự báo của mô hình. Bùi Hoàng Hải và Phan Văn Tân (2002) [1] khảo sát ảnh hưởng quá trình ban đầu hóa tới quỹ đạo dự báo bằng việc chạy mô hình WBAR cho 3 cơn bão Durian (2001), Kajiki (2001), Wukong (2000). Kết quả cho thấy ban đầu hóa xoáy đã góp phần làm giảm sai số vị trí của quỹ đạo dự báo. Phan Văn Tân và Nguyễn Lê Dũng (2008) [15] đã xây dựng xoáy nhân tạo bằng cách đồng hóa số liệu quan trắc giả từ mô-đun đồng hóa dữ liệu biến phân ba chiều 3D-VAR trong mô hình WRF thử nghiệm dự báo đối với 10 cơn bão hoạt động trên khu vực biển Đông trong khoảng thời gian từ 20062008. Kết quả nghiên cứu cho thấy, việc sử dụng bộ số liệu "giả" đã cải thiện đáng kể chất lượng dự báo quỹ đạo bão, nhất là đối với các cơn bão mạnh. Trần Tân Tiến và Lê Thị Hồng Vân (2009) [17] nghiên cứu sự ảnh hưởng của các yếu tố cấu thành xoáy nhân tạo trong đồng hóa số liệu xoáy giả bằng mô hình WRF đối với cơn bão Lekima đã nhận định rằng vai trò của ban đầu hóa xoáy là quan trọng trong cải thiện chất lượng dự báo bão, đặc biệt là về cường độ. Dư Đức Tiến và cộng sự (2016) [3] đã sử dụng đồng thời thông tin quan trắc gió tại các mực trên cao tính toán từ sự dịch chuyển của mây do Trường đại học Wisconsin, Hoa kỳ cung cấp để xây dụng mô hình xoáy ba chiều đầy đủ bằng phương pháp đồng hóa tổ hợp lọc Kalman (LetKF) cho mô hình WRF-ARW. Nghiên cứu được thực hiện thử nghiệm trên cơn bão Usagi (2013) hoạt động trên khu vực Tây Bắc Thái Bình Dương. Kết quả cho thấy việc đưa thông tin quan trắc vào góp phần tăng cường chất lượng dữ báo quỹ đạo được cải thiện tại hầu hết các hạn dự báo.

Các nghiên cứu trước đây cho thấy ban đầu hóa xoáy có nhiều ưu điểm vượt trội so với trường hợp không có ban đầu hóa. Câu hỏi đặt ra là, nếu với ban đầu hóa xoáy động lực, mô hình WRF có thể dự báo được tốt quỹ đạo và cấu trúc, sản phẩm mô hình có thể sử dụng để nghiên cứu ảnh hưởng của địa hình và hoàn lưu quy mô lớn tới cấu trúc bão Mujigae (2015). Xuất phát từ thực tế trên, bài báo này sẽ sử dụng mô hình WRF với ban đầu hóa xoáy động lực để nghiên cứu cấu bão Mujigae (2015) giai đoạn bão gần bờ và đổ bộ. Phần tiếp theo của bài báo mô tả số liệu và phương pháp nghiên cứu ở Mục 2 . Mục 3 là Kết quả. Cuối cùng phần kết luận ở Mục 4.

\section{Số liệu và phương pháp nghiên cứu}

\subsection{Số liệu}

Trong nghiên cứu này, bộ số liệu chỉ thị của bão (besttrack) cung cấp bởi của Cơ quan Khí tượng Nhật Bản (JMA) và của Trung tâm cảnh báo bão Hoa kỳ (JTWC) được sử dụng làm số đầu vào cho chương trình ban đầu hóa xoáy. Số liệu besttrack bao gồm: khu vực bão hình thành, vị trí tâm bão (vĩ độ và kinh độ) và cường độ (tốc độ gió mạnh nhất, khí áp mực biển thấp nhất cách nhau 6 giờ. Số liệu trường ban đầu và điều kiện biên phụ thuộc thời gian được lấy từ sản phẩm phân tích FNL của Trung tâm Dự báo Môi trường Hoa kỳ (NCEP).

\subsection{Phuơng pháp nghiên cứu}

a) Phuoong pháp ban đầu hóa xoáy động lục

Nghiên cứu này ứng dụng phương pháp ban đầu hóa xoáy động lực NC2011 của nhóm tác 
giả Nguyễn Văn Hiệp và Yi-Leng Chen (2011) [5] để tạo một trường ban đầu cho mô hình WRF nhằm thử nghiệm dự báo cường độ cơn bão $\mathrm{Mu}$ jigae năm 2015. Đây là phương pháp ban đầu hóa thông qua kỹ thuật chạy lặp nhằm tạo điều kiện ban đầu độ phân giải cao cho mô hình số. Xoáy bão được tạo ra có cấu trúc phi đối xứng ba chiều phù hợp động lực với điều kiện môi trường bão Nguyen và Chen (2011) [7]. Cường độ, kích thước và vị trí bão phù hợp với thực tế. Chương trình ban đầu hóa xoáy động lực NC2011 được xây dựng với hai giả thiết được sử dụng: thứ nhất là trong khoảng thời gian ngắn (dưới 1 giờ) XTNĐ di chuyển nhưng cấu trúc của nó thay đổi không đáng kể; thứ hai là cấu trúc của XTNĐ được khống chế bởi trường môi trường bão. Trên cơ sở đó, sơ đồ NC2011 xây dựng có các đặc điểm cơ bản như: Sử dụng dữ liệu dự báo toàn cầu làm là điều kiện ban đầu cho quá trình khởi tạo xoáy thông qua khoảng 60-80 vòng lặp với thời gian tích phân mỗi vòng lặp là 1 giờ. Sau mỗi vòng lặp, xoáy từ vòng lặp trước đó được tách ra và sử dụng làm xoáy ban đầu cho vòng lặp tiếp trong khi trường môi trường được giữ

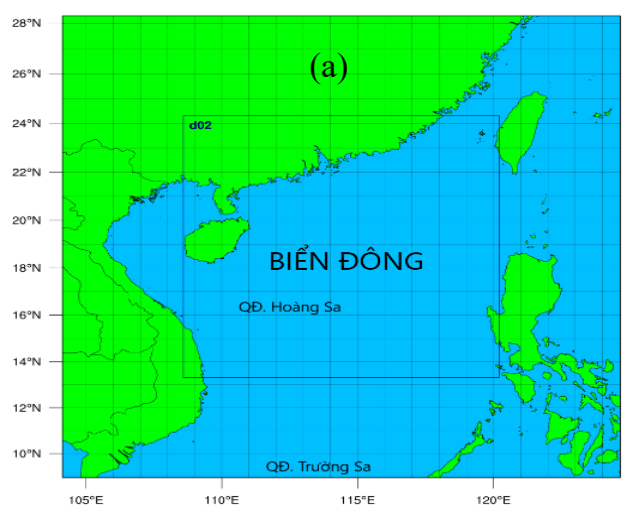

Hình 1. Miền tính sủ dụng trong quá trình chạy ban đầu hóa xoáy (a) và mô phỏng (b)

\section{Kết quả}

\section{1 Điều kiện hình thành và hoạt động bão}

\section{Mujigae}

Bão Mujigae hay còn gọi là cơn bão số 4 năm 2015 được hình thành từ một vùng áp thấp nhiệt đới trên vùng biển phía đồng quần đảo Phi-lippin. Theo Cơ quan Khí tượng Nhật Bản, vào 18 giờ ngày 30/9/2015, cách $160 \mathrm{~km}$ về phía đông hòn đảo Samar Phi-lip-pin tồn tại một trung tâm áp thấp. Sau 12 giờ vùng thấp này di chuyển theo không đổi [5].

\section{b) Thiết kế thí nghiệm}

Trong nghiên cứu này sử dụng mô hình Nghiên cứu và Dự báo thời tiết WRF (Weather Research and Forecast) của Hoa Kỳ. Đây là mô hình được phát triển từ những đặc tính ưu việt nhất của mô hình MM5 với sự cộng tác của nhiều cơ quan tổ chức lớn trên thế giới. Hiện nay, mô hình WRF đang được sử dụng rộng rãi trong dự báo thời tiết nghiệp vụ cũng như trong nghiên cứu ở nhiều quốc gia trên thế giới.

Các lựa chọn vật lý của mô hình tương tự như Nguyen and Chen (2011) [7]. Miền tính được sử dụng để chạy ban đầu hóa xoáy và mô phỏng bao gồm 2 lưới lồng ghép nhau với độ phân giải lần lượt là $18 \mathrm{~km}$ và $6 \mathrm{~km}$. Trong đó, kích thước miền tính sử dụng chạy ban đầu hóa lần lượt là 121x121, 205x205 điểm lưới. Miền tính chạy mô phỏng có kích thước lớn hơn, 301x221 và 385x337 điểm lưới, để có thể tính toán được ảnh hưởng của các hình thế thời tiết khống chế khu vực (Hình 1). Cần chú ý rằng, trong phương pháp NC2011, vị trí các miền tính tại mỗi thời điểm mô phỏng được xác định tự động theo tâm bão.

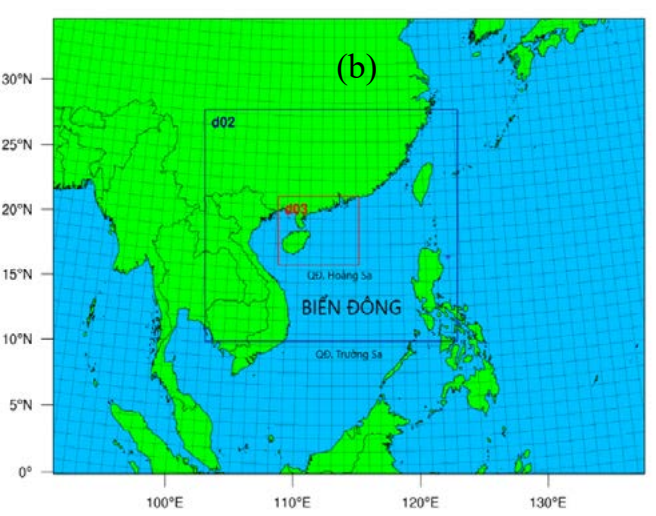

hướng tây-tây bắc và mạnh lên thành một áp thấp nhiệt đới. Tới thời điểm 12 giờ ngày 01/10/2015, áp thấp nhiệt đới mạnh lên thành bão và được đặt tên là Mujigae. Đến thời điểm 04 giờ ngày 02/10/2015, bão Mujigae đổ bộ lần thứ nhất vào tỉnh Aurora của Phi-lip-pin, vị trí tâm bão ở khoảng 16,1 độ vĩ Bắc và 121 độ kinh đông và giảm cấp thành một áp thấp nhiệt đới.

Sau khi rời khỏi quần đảo Phi-lip-pin vào $\mathrm{khu}$ vực biển Đông, nơi có độ đứt gió yếu và nhiệt 
độ bề mặt nước biển cao góp phần tạo điều kiện cho áp thấp mạnh lên thành bão. Trong 24 giờ tiếp theo, bão tiếp tục di chuyển theo hướng tây tây bắc về phía tây Quảng Đông - Trung Quốc. Đến thời điểm 00 giờ ngày $04 / 10 / 2015$ khi cách Hồng Kông khoảng $350 \mathrm{~km}$ về phía nam tây nam, cường độ của bão mạnh lên đột ngột với vận tốc gió cực đại tại tâm lên tới trên $50 \mathrm{~m} . \mathrm{s}-1$ và đổ bộ lần thứ hai vào Trạm Giang, Trung Quốc vào thời điểm $06 \mathrm{Z}$ ngày $04 / 11 / 2015$, sau đó tan giã vào khoảng $00 Z$ ngày $05 / 10 / 2015$.

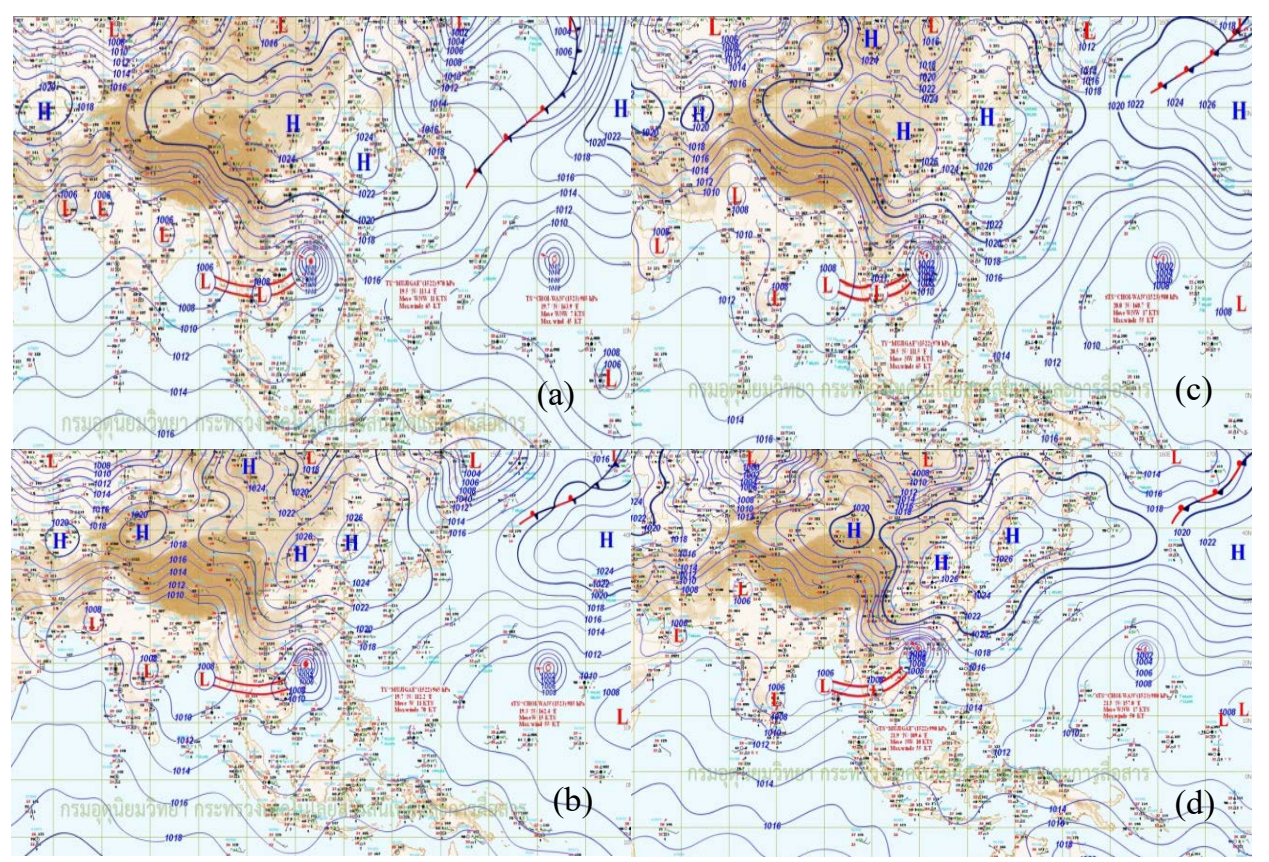

Hình 2. Hình thế synop trong thòi gian bão Mujigae hoạt động (a) 12Z 03/10, (b) 18Z 03/10, (c) $00 Z$ 04/2015 và (d) $12 Z$ 04/10/2015 (nguồn: www.tmd.go.th)

Về hình thế synop, tại thời điểm 12UTC ngày 03 tháng 10 năm 2015, khu vực phía Bắc Việt Nam chịu tác động của áp cao lạnh lục địa lệch đông có cường độ yếu, bão Mujigae nằm trên một rãnh thấp đi qua Biển Đông và Ân Độ Dương. Đến thời điểm $12 \mathrm{UTC}$ ngày 04 tháng 10 năm 2015, khu vực phía bắc của bão tiếp tục chịu tác động của áp cao lạnh lục địa lệch đông có cường độ tăng dần cho tới khi bão Mujigae đổ bộ vào bán đảo Lôi Châu với cường độ khá mạnh khi đi vào đất liền (Hình 2).

\subsection{Vai trò của ban đầu hóa xoáy động lục}

Kỹ thuật ban đầu hóa xoáy được trình bày trong phần 2 được kết hợp với mô hình WRF phiên bản 3.7. Trong phần này trình bày kết quả mô phỏng hạn 24 giờ bão Mujigae (2015) với thời điểm ban đầu lúc $06 \mathrm{Z}$ ngày $03 / 10 / 2015$. Đây là thời điểm 24 giờ trước khi bão đổ bộ vào đất liền khu vực bán đảo Nam Trung Quốc. Hai thí nghiệm được thực hiện bao gồm: (1) điều kiện ban đầu được lấy thuần túy từ phân tích toàn cầu FNL của NCEP (CTRL) và (2) điều kiện ban đầu được cập nhật với sơ đồ ban đầu hóa xoáy NC2011 (Bogus).

Trong giả thiết xây dựng sơ đồ ban đầu hóa xoáy động lực của NC2011 của Nguyễn Văn Hiệp và Yi-Leng Chen (2011) [5], một giả thiết mặc định là giá trị quan trắc vị trí và cường độ bão có chất lượng tốt. Trên cơ sở đó khi mô hình khởi tạo (spin-up), sau một số vòng lặp nhất định, cường độ xoáy bão mô hình sẽ tiến tới cường độ xoáy bão quan trắc, các biến trong mô hình cũng đạt trạng thái tựa cân bằng giữa các quá trình quy mô vừa trong xoáy bão với trường môi trường quy mô lớn. Do vậy, trước khi áp dụng sơ đồ NC2011, chất lượng cường độ xoáy bão quan trắc cần được xem xét. Hình 3 cho thấy sự sai khác lớn nhất trong số liệu besttrack đối với khí áp cực tiểu tại tâm bão Mujigae giữa hai trung tâm JMA và JTWC khoảng 5-8 hPa. Sai 
khác lớn nhất tại các thời điểm bão đang phát triển (trước $06 Z$ ngày $03 / 10$ ) và khi bão sát bờ và đổ bộ (sau $00 Z$ ngày 4/10/2015) (Hình 3a). Sai khác với gió cực đại từ sau $00 \mathrm{Z}$ ngày $4 / 10 / 2015$ là lớn. Sai khác lớn nhất vào $06 Z$ ngày 4/10/2015 với giá trị tới $11 \mathrm{~m} \mathrm{~s}-1$ (Hình 3b). Độ lớn của sai khác này lớn hơn sai số trung bình dự báo cường độ hạn $24 \mathrm{~h}$ của nhiều trung tâm lớn trên thế giới. Đây là một ví dụ điển hình về chất lượng số liệu số liệu quan trắc cường độ bão khi được xác định chủ yếu qua các công thức thực nghiệm từ các trường quan trắc vệ tinh. Ngoài một số cơn bão có số liệu thám sát máy bay, số liệu besttrack ước lượng từ số liệu vệ tinh hiện nay là nguồn số liệu chính sử dụng trong đánh giá chất lượng dự báo cường độ bão. Khi sử dụng số liệu này để hiệu chỉnh mô hình và đánh giá chất lượng dự báo, nếu chất lượng số liệu kiểm chứng không tốt sẽ dễ dẫn tới mất phương hướng trong nghiên cứu và dự báo cường độ bão. Mức độ chưa chắc chắn cao trong số liệu quan trắc cường độ bão có thể là một trong những nguyên nhân quan trọng làm chất lượng dự báo cường độ bão không của cộng đồng khí tượng thế giới không có những biến chuyển đáng kể trong mấy chục năm gần đây.
Trong khuôn khổ bài báo này, do số liệu quan trắc cường độ bão Mujigae có mức độ chưa chắc chắn cao, khi áp dụng phương pháp đầu hóa xoáy động lực của NC2011 của Nguyễn Văn Hiệp và Yi-Leng Chen, chúng tôi không sử dụng tiêu chí nguyên gốc là so sánh sai khác cường độ để dừng quá trình khởi tạo. Thay vào đó, quá trình khởi tạo được dừng khi cấu trúc mây trong bão, cụ thể là cấu trúc trường độ phản hồi radar, có mức độ tương tự tương đối với cấu trúc mây đối lưu sâu trên ảnh mây vệ tinh Himawari 8 .

Độ phản hồi vô tuyến của bão tại thời điểm ban đầu cho hai trường hợp trước và sau ban đầu hóa xoáy (Hình 3) cho thấy khi không ban đầu hóa xoáy, phản hồi vô tuyến mô phỏng (Hình 3a) không phù hợp với quan trắc vệ tinh. Sau ban đầu hóa xoáy (Hình 3b) hình dạng của bão khá tương đồng với ảnh mây vệ tinh (Hình 3c), đặc biệt vùng gần tâm bão. Cấu trúc phi đối xứng của bão thể hiện rõ trên số liệu vệ tinh và mô phỏng mô hình sau khi ban đầu hóa xoáy, với hai khu vực mây đối lưu sâu phát triển mạnh là phía nam và phía đông bắc mắt bão. Khu vực này có độ phản hồi tương đối cao, đạt từ 45-50dbz (Hình 3 b), trùng với vùng có mây đối lưu sâu trên ảnh mây vệ tinh (Hình 3c).
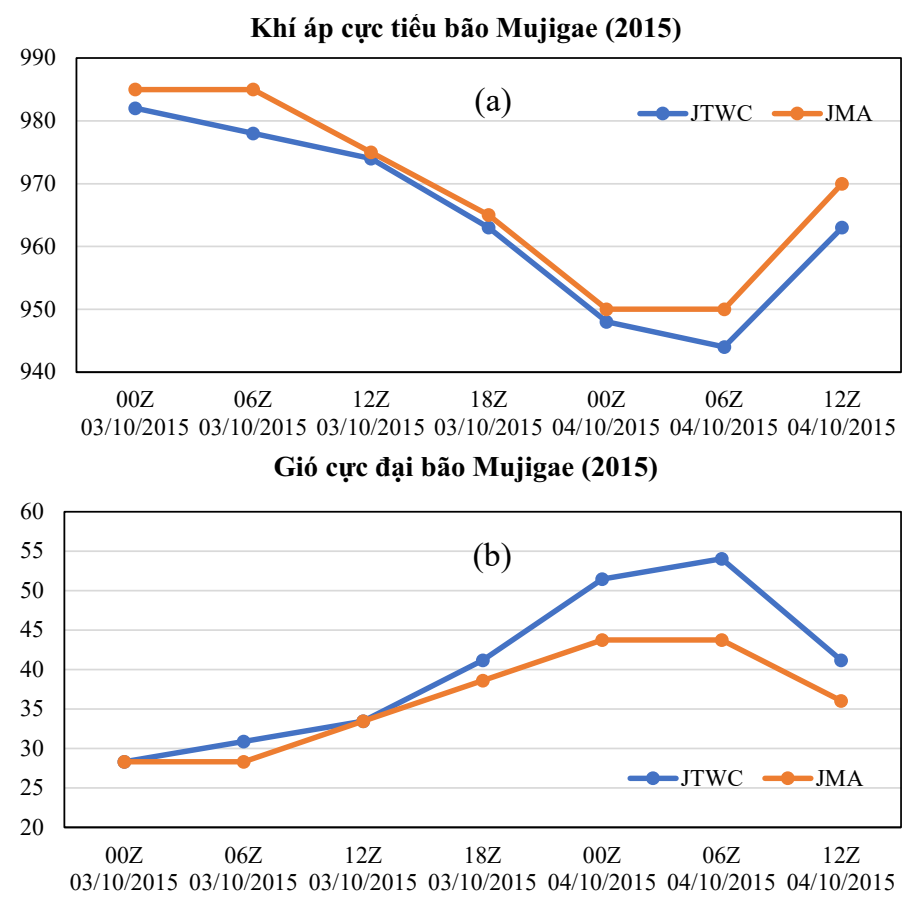

Hình 3. Biến trình (a) khi áp cực tiểu ( $h P a$ ) và (b) tốc độ gió cực đại (m.s-1) bão Mujigae (2015) tù̀ nguồn JTWC (màu xanh) và JMA (màu đỏ) 
(a)

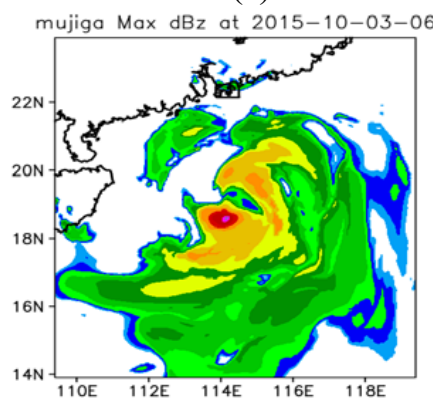

(b)

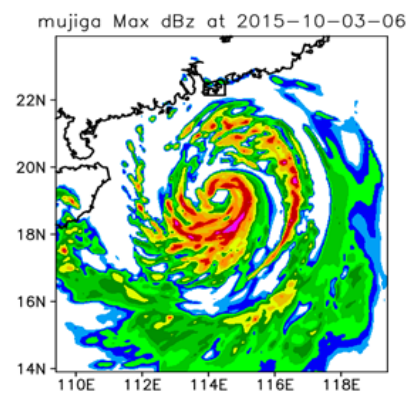

(c)

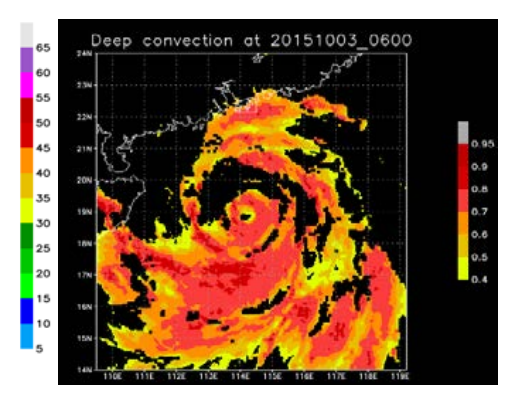

Hình 4. Độ phản hồi vô tuyến tại thời điểm ban đầu 06Z ngày 03/10/2015 trương hợp (a) không ban đầu hóa xoáy, (b) có ban đầu hóa xoáy và (c) vùng đối lưu sâu xác định tù kênh thị phổ của vệ tinh Himawari 8 cùng thời điểm

Mặt cắt dọc vĩ tuyến qua tâm bão trên Hình 5 cho thấy gió cực đại trên trường phân tích (chưa có ban đầu hóa xoáy) là $31 \mathrm{~m} \mathrm{~s}-1$, tương đương với số liệu JTWC nhưng mạnh hơn số liệu JMA. Khí áp cực tiểu phân tích chỉ đạt $995 \mathrm{hPa}$, cao hơn nhiều so với giá trị của JMA là $985 \mathrm{hPa}$ và JTWC là 978 hPa (Hình 5). Sau khi ban đầu hóa xoáy trường khí áp mực biển cực tiểu được khơi sâu và tốc độ gió cực đại được tăng cường. Khi mô hình tái tạo được cấu trúc mây phù hợp quan trắc vệ tinh (Hình 4), giá trị áp cực tiểu đạt 972 hPa, gió cực đại đạt 36m s-1 (Hình 5).

Để tiếp tục phân tích sự khác biệt giữa trường ban đầu trước và sau khi ban đầu hóa xoáy, mặt cắt vĩ hướng qua tâm bão đối với trường tốc độ gió $(\mathrm{m} \mathrm{s}-1)$ và dị thường nhiệt độ $(\mathrm{K})$ cho hai trường hợp không có ban đầu hóa xoáy và có ban đầu hóa xoáy được đưa ra trên Hình 5. Kết quả cho thấy dị thường nhiệt độ trong trường phân tích đạt cực đại khoảng 6-8K tại độ cao khoảng $8 \mathrm{~km}$ (Hình 6a). Sau khi ban đầu hóa xoáy, dị thường nhiệt độ đạt cực đại ở độ cao khoảng 10 $\mathrm{km}$ và giá trị tăng lên tới $8-10 \mathrm{~K}$. Sự tăng lên của dị thường nhiệt độ này phù hợp với cường độ bão tăng lên gần với giá trị cường độ quan trắc. Trên thực tế bão càng mạnh thì áp cực tiểu tại tâm giảm và dị thường nhiệt độ mực cao vùng tâm bão tăng.

Hình 6 cũng cho thấy sau khi ban đầu hóa xoáy, phân bố cường độ gió vùng gần tâm bão cũng phù hợp hơn. Trong khi bán kính gió cực đại trên trường phân tích tới khoảng $100 \mathrm{~km}$ (Hình $6 \mathrm{a}$ ), bán kính này trên trường ban đầu hóa xoáy khoảng $50 \mathrm{~km}$ (Hình 6b). Vậy độ lớn bán kính gió cực đại trong trường sau khi ban đầu hóa xoáy phù hợp với thực tế hơn. Bán kính mắt bão thực tế ước lượng từ ảnh mây vệ tinh (Hình $4 \mathrm{c})$ trong trường hợp này cũng khoảng $50 \mathrm{~km}$.

Với trường hợp bão Mujigae, không có sự khác biệt lớn đối với số liệu quan trắc quỹ đạo. Về chất lượng mô phỏng quỹ đạo, cả hai trường hợp có và không có ban đầu hóa xoáy đều cho kết quả mô phỏng khá tốt quỹ đạo bão. Mặc dù vậy, trừ thời điểm mô phỏng 24h ban đầu hóa xoáy cho kết quả quỹ đạo tốt hơn trường hợp không ban đầu hóa xoáy. Sau khi ban đầu hóa xoáy, sai số quỹ đạo của tất cả các hạn mô phỏng đều nhỏ hơn $50 \mathrm{~km}$. Với cấu trúc mây phù hợp với ảnh mây vệ tinh, vị trí mô phỏng gần như không sai khác với quan trắc, sản phẩm mô phỏng của mô hình có thể sử dụng để nghiên cứu sự biến đổi cấu trúc ba chiều của bão khi gần bờ và đổ bộ. 

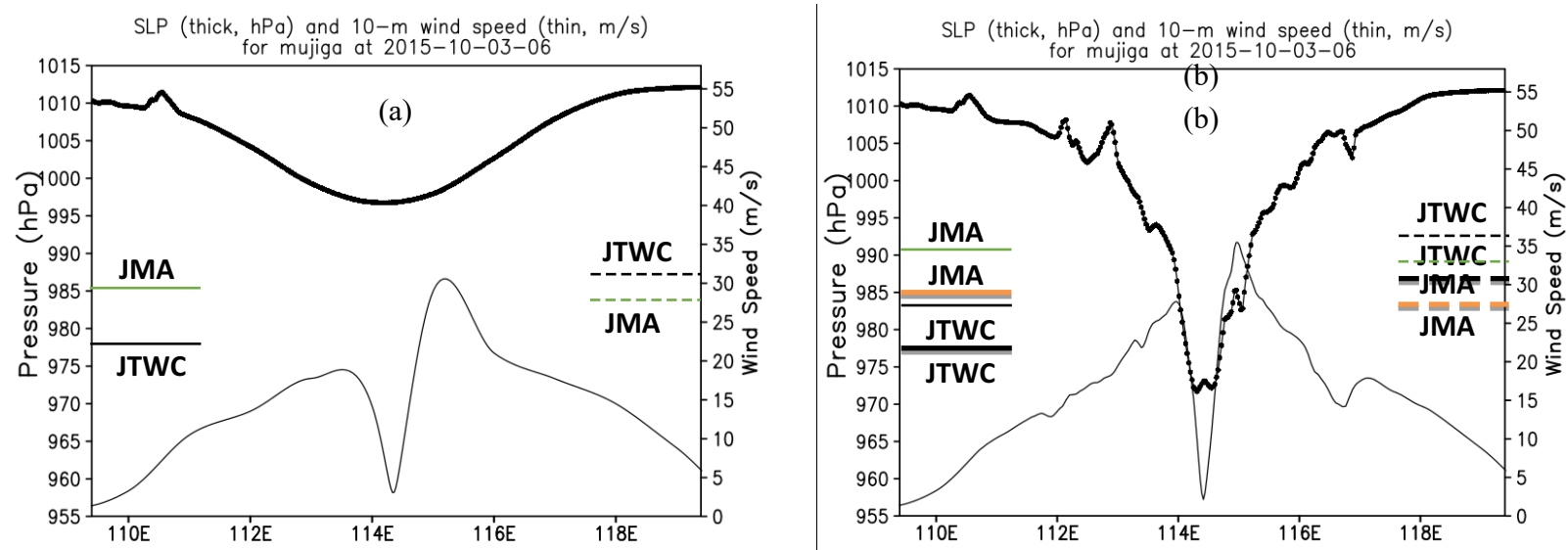

Hình 5. Mặt cắt vĩ hướng qua tâm bão truoòng gió mục $10 \mathrm{~m}$ (m.s-1) và khí áp mưc mặt biển (hPa) bão Mujigae cho truờng hợp (a) không có ban đầu hóa xoáy và (b) có ban đầu hóa xoáy. Các đuờng kẻ ngang đánh dấu giá trị quan trắc khí áp cưc tiểu (gió cục đại) của JTWC và của JMA tại thời điểm 00Z ngày 03/10/2015

(a)

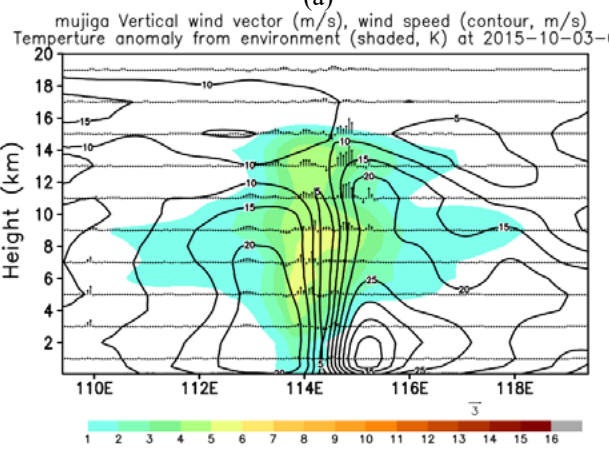

(b)

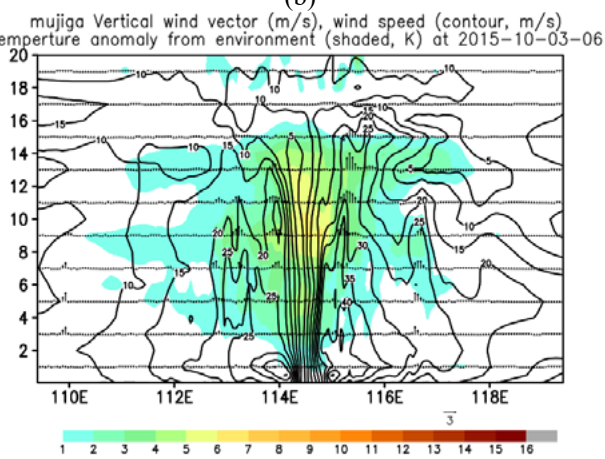

Hình 6. Mặt cắt vĩ hướng qua tâm bão truờng tốc độ gió ( $m s-1)$ và dị thuờng nhiệt độ (K) bão

Mujigae cho trường hợp (a) không có ban đầu hóa xoáy và (b) có ban đầu hóa xoáy

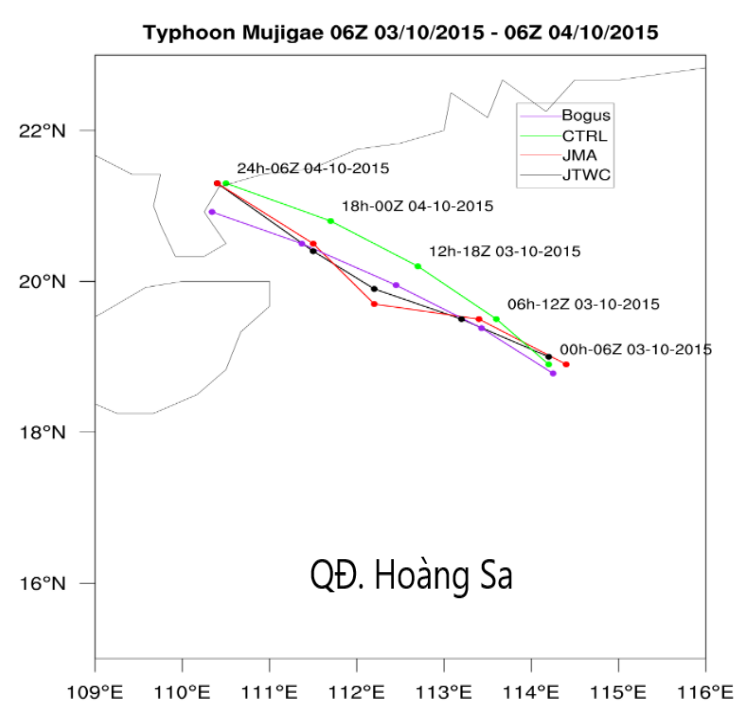

Hình 7. Quỹ đạo quan trắc bão Mujigae (2015) của JMA (đỏ), JTWC (đen) và mô phỏng với có ban đầu hóa xoáy (tím) và không ban đầu hóa xoáy (xanh)

\subsection{Cấu trúc bão Mujigae (2015) khi gần bò̀ và đổ bọ}

Trên cơ sở mô phỏng tốt quỹ đạo và cấu trúc mây, sản phẩm mô hình chạy với ban đầu hóa xoáy được sử dụng để đưa ra các nhận định về cấu trúc thẳng đứng của bão giai đoạn gần đảo bờ và đổ bộ. Hình 8 biểu diễn độ phản hồi vô tuyến mô phỏng 6 giờ một. Tại các thời điểm $12 Z 03 / 10 / 2015$, và $18 Z$ Z 03/201/2015 khi tâm bão còn cách đất liền trên $100 \mathrm{~km}$, phân bố độ phản hồi radar vùng gần tâm bão khá đối xứng. Vùng xa tâm bão, đối lưu sâu chủ yếu phát triển khu vực trên biển phía nam và đông nam so với tâm bão. Khi bão đổ bộ, vùng $300 \mathrm{~km}$ từ tâm bão có tính phi đối xứng mạnh với vùng mây đối lưu sâu tập trung về phía bắc tâm bão (phía đất liền) có thể do tương tác hoàn lưu bão với địa hình và 


\section{BÀI BÁO KHOA HỌC}

đất liền nơi có ma sát lớn.

Để nghiên cứu cấu trúc của bão khi gần bờ, các Hình 9 và 10 mô tả mặt cắt thẳng đứng qua tâm bão tại các thời điểm tương ứng với trên Hình 8 . Hình 9 cho thấy vùng bán kính khoảng $100 \mathrm{~km}$ gần tâm bão, tốc độ gió mực thấp dưới $2 \mathrm{~km}$ tương đối đối xứng. Ở trên cao (trên $2 \mathrm{~km}$ ) gió mạnh (tốc độ gió $>35 \mathrm{~m} \mathrm{~s}-1$ ) chủ yếu tập trung phía đông của tâm bão. Vùng xa hơn của tâm bão (bán kính từ 150 tới 400km), gió mạnh cũng chủ yếu tập trung ở phía đông của tâm bão (Hình 9). Vùng gió mạnh phía đông này có thể do các nguyên nhân chính như: (1) đối lưu khu vực này mạnh (Hình 8 ) giúp mang các phần tử khí có động năng lớn mực thấp lên cao hơn, (2) gió gradient tăng cường do tương tác giữa hoàn lưu bão với cao lạnh lục địa phía bắc cơn bão và (3) của ma sát trên biển nhỏ. Cần chú ý rằng khi tâm bão mô phỏng bão đổ bộ vào $06 Z$ 04/10/2015, phần phía đông của hoàn lưu bão vẫn nằm trên biển.

Mặt cắt thẳng đứng-bắc nam (Hình 10) cũng thể hiện rõ phân bố phi đối xứng của trường gió. Thông thường phần phía nam của cơn bão do ở trên biển có ma sát nhỏ phải có gió mạnh hơn phía bắc của cơn bão, trong trường hợp này tình hướng lại ngược lại, phía bắc tâm bão (phần trên đất liền) lại có gió mạnh hơn (Hình 10). Sự bất thường này có thể do gió phần phía bắc cơn bão tăng cường do gió gradient mạnh khi cao lạnh lục địa phía bắc hoạt động.
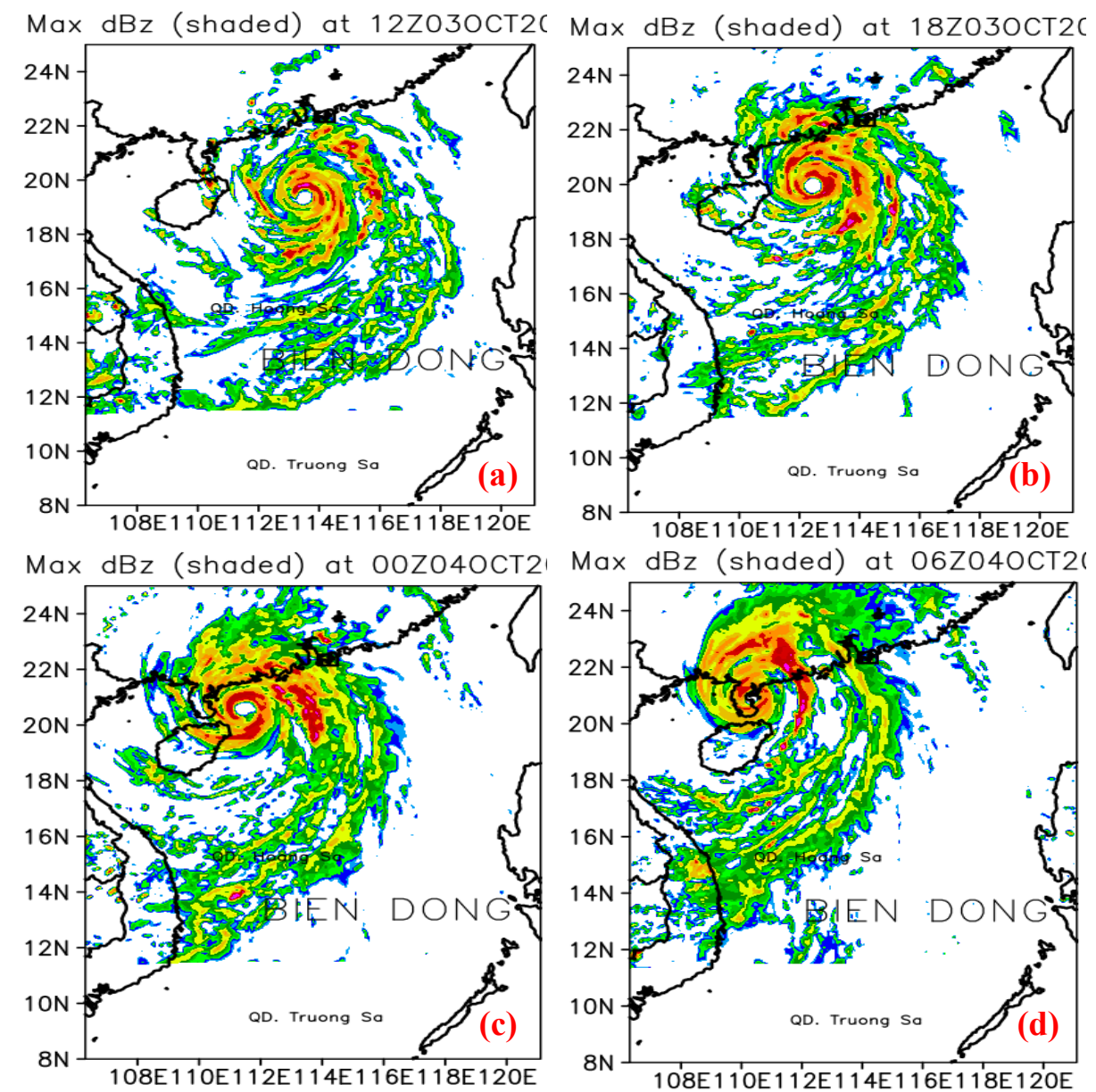

Hình 8. Độ phản hồi vô tuyến mô phỏng tại (a) 12Z 03/10/2015, (b) 18Z 03/10/2015, (c) $00 Z$ 04/10/2015 và 06Z 04/10/2015 tù truòng hợ có ban đầu hóa 
(a)

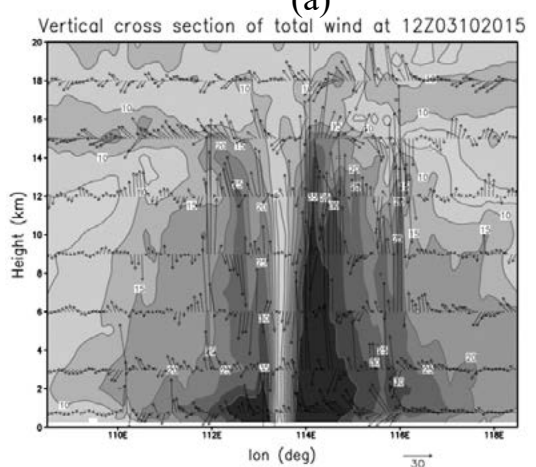

(c)

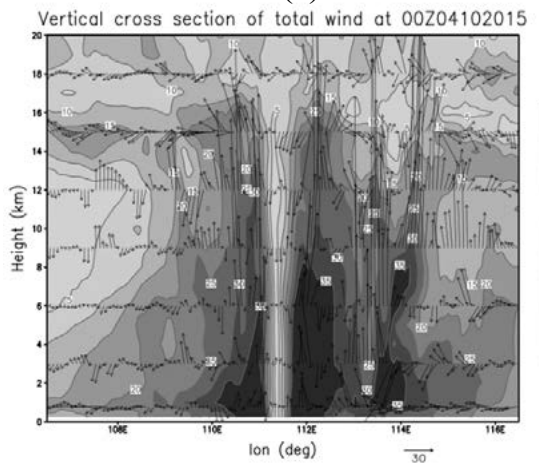

(b)

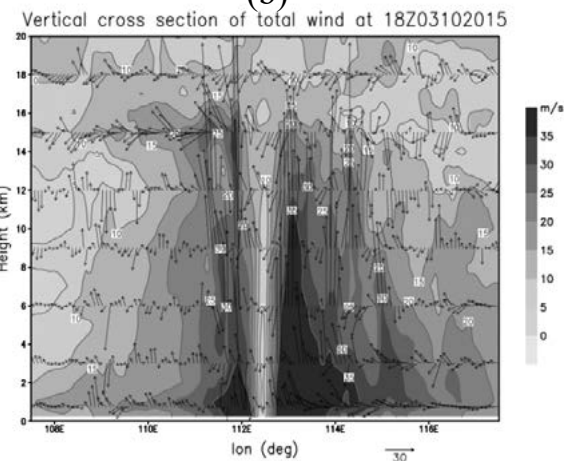

(d)

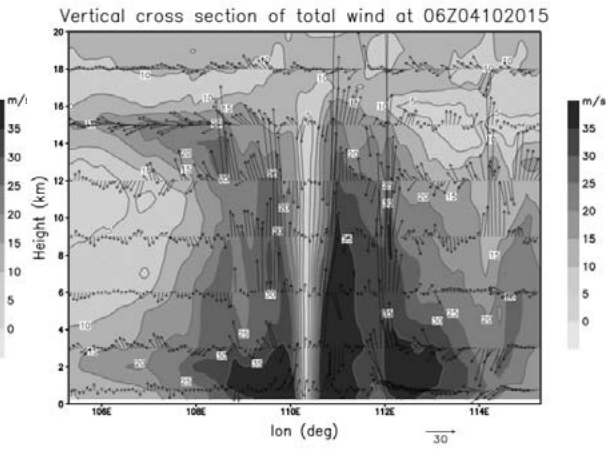

Hình 9. Mặt cắt thẳng đí̛ng-đông tây qua tâm bão đối với trương gió (m.s-1) mô phỏng có ban đầu hóa tại (a) 12Z 03/10/2015, (b) 18Z 03/10/2015, (c) 00Z 04/10/2015 và (d) 06Z 04/10/2015

(a)

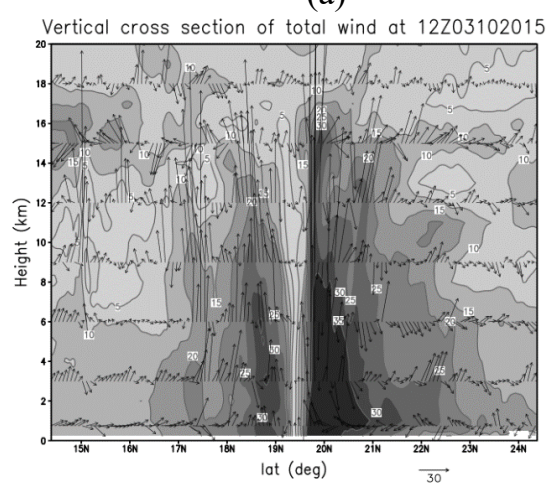

(c)

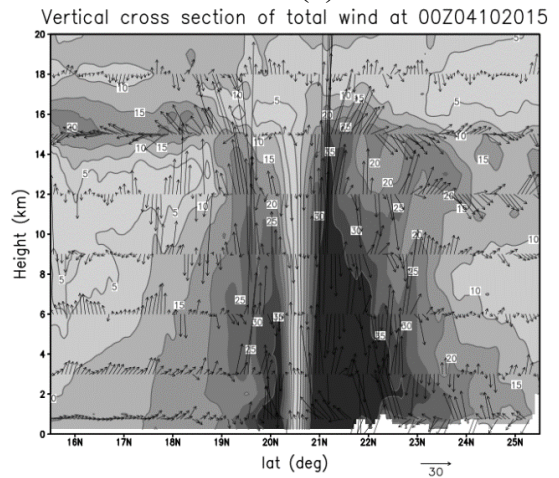

(b)

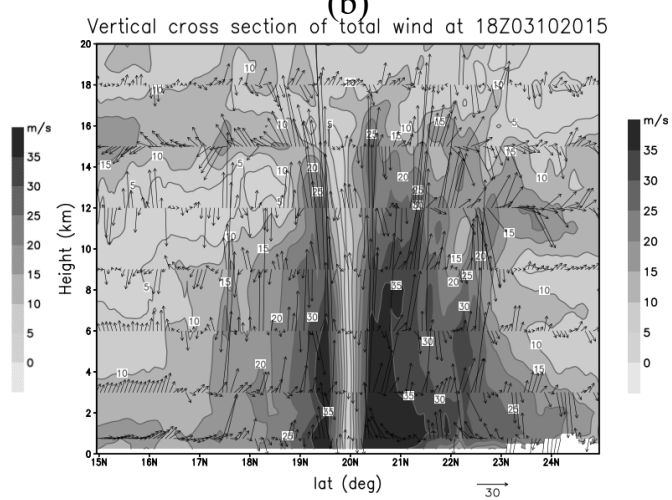

(d)

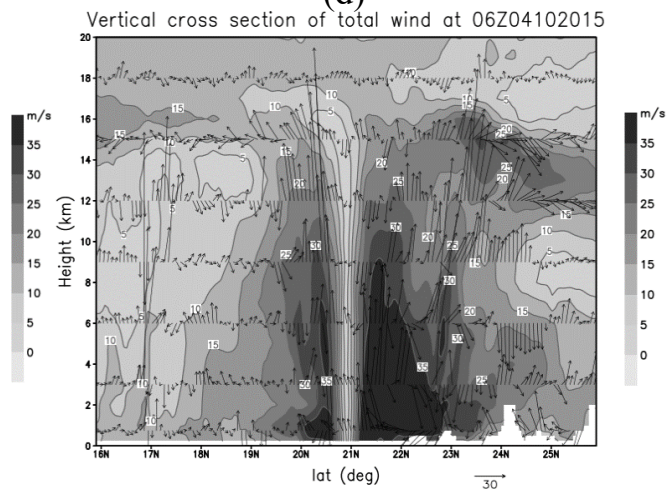

Hình 10. Mặt cắt thẳng đứng-nam bắc qua tâm bão đối với trừ̀ng gió mô phỏng có ban đầu hóa tại (a) 12Z 03/10/2015, (b) 18Z 03/10/2015, (c) 00Z 04/10/2015 và (d) 06Z 04/10/2015 


\section{BÀI BÁO KHOA HỌC}

\section{Kết luận}

Bài báo đã ứng dụng sơ đồ ban đầu hóa xoáy động lực của Nguyễn Văn Hiệp và Yi-Leng Chen xây dựng năm 2011 có cải tiến, kết hợp với WRF (Weather Research Forecasting) để nghiên cứu ảnh hưởng của địa hình và gió mùa đông bắc tăng cường bởi cao lạnh lục địa tới cấu trúc bão Mujigae (2015). Quan trắc cường độ bão cho thấy có sự khác biệt đáng kể (tới $10 \mathrm{~m} \mathrm{~s}-1$ ) giữa số liệu tại JTWC và JMA. Sự chưa chắc chắn cao trong quan trắc cường độ bão có thể là một trong những nguyên nhân quan trọng dẫn đến chất lượng dự báo cường độ bão trên thế giới gần như không có cải thiện đáng kể trong mấy chục năm gần đây. Do mức độ chưa chắc chắn cao trong số liệu quan trắc cường độ bão Mujigae, việc ứng dụng chỉ tiêu nguyên thủy trong sơ ban đầu hóa xoáy động lực của NC2011 không phù hợp. Lý do là khó xác định được trạng thái cân bằng động lực của xoáy bão dựa trên sự sai khác giữa cường độ xoáy khởi tạo với cường độ quan trắc nếu sô liệu quan chắc không chính xác. Do vậy, khi áp dụng phương pháp NC2011 trong bài báo này, tiêu chí áp dụng thay thế để dừng quá trình khởi tạo là khi cấu trúc trường độ phản hồi radar có mức độ tương tự tương đối với cấu trúc mây đối lưu sâu trên ảnh mây vệ tinh Himawari 8 .

Sau khi ban đầu hóa xoáy, trường khí áp mực biển cực tiểu được khơi sâu và tốc độ gió cực đại được tăng cường. Mô hình tái tạo được cấu trúc mây đối lưu sâu phù hợp thực tế, phân bố cường độ gió vùng gần tâm bão cũng phù hợp hơn, bán kính mắt bão gần với thực tế hơn, sai số quỹ đạo của tất cả các hạn mô phỏng tới $24 \mathrm{~h}$ đều nhỏ hơn $50 \mathrm{~km}$.

Phân tích sản phẩm mô hình chạy với ban đầu hóa xoáy cho thấy cấu trúc hoàn lưu bão Mujigae có tính phi đối xứng mạnh. Khi bão trên biển, vùng gió mạnh hơn nằm ở phía đông có thể do các nguyên nhân chính như: (1) đối lưu khu vực này mạnh giúp mang các phần tử khí có động năng lớn mực thấp lên cao hơn, (2) gió gradient tăng cường do tương tác giữa hoàn lưu bão với cao lạnh lục địa phía bắc cơn bão và (3) ma sát trên biển nhỏ. Khi bão đổ bộ, điểm khác biệt trong cơn bão Mujigae là phần trên đất liền phía bắc nơi có ảnh hưởng mạnh của ma sát trên đất liền lại có gió mạnh hơn phần trên biển nơi có ảnh hưởng ma sát yếu. Sự bất thường này có thể do gió phần phía bắc cơn bão được tăng cường do gió gradient mạnh khu vực này khi có sự hoạt động của cao lạnh lục địa phía bắc.

Lời cảm ơn: Bài báo này được thực hiện trong khuôn khổ thực hiện dụ án "Thiết lập hệ thống quan trắc tăng cường và hệ thống dụ báo, cảnh báo độ phân giải cao hạn ngắn, cực ngắn dông, mưa lớn và ngập lụt đô thị cho thành phố Hà Nội phục vu phát triển kinh tế, đảm bảo an sinh xã họi", số TTTT: 16/FIRST/2a/IGP thuộc Tiểu hợp phần 2a, Dự án FIRST. Nghiên cứu này cũng được hố trọ" bởi đề tài "Nghiên cúu vai trò của địa hình và cuõong búc động lực trong cơ chế hình thành mưa lớn khu vực Việt Nam bằng mô hình số trị WRF (Weather Research and Forecasting Model)" Mã số: 105.06-2016.12 do Quỹ NAFOSTED tài trợ.

\section{Tài liệu tham khảo}

1. Bùi Hoàng Hải, Phan Văn Tân (2002), Khảo sát ảnh hương của trường ban đầu hóa đến sự chuyển động của bão trong mô hình chính áp dụ báo quĩ đạo bão khu vục Biển Đông. Tạp chí Khí tượng Thủy Văn, 500, 17-23.

2. Chan, J.C.L., Williams, R.T., (1987), Analytical and numerical study of the beta-effect in tropical cyclone motion. Part I: Zero mean flow. Journal of the Atmospheric Sciences, 44, 1257-1265.

3. Chou, K.H., Wu, C.C., Lin, P.H., Majumdar, S., (2010), Validation of QuikSCAT wind vectors by dropwindsonde data from Dropwindsonde Observations for Typhoon Surveillance Near the Taiwan Region (DOTSTAR), Journal of Geophysical Research, 115. 
4. Chou, K.H., Wu, C.C., (2008), Typhoon Initialization in a Mesoscale Model - CohPaination of the Bogused Vortex and the Dropwindsonde Data in DOTSTAR, Monthly Weather Review 136(3), 865-879.

5. Dư Đức Tiến, Ngô Đức Thành, Kiều Quốc Chánh (2016), Sư dụng đồng thời quan trắc quy mô lớn và quy mô bão trong việc tăng cuờng thông tin ban đầu cho bài toán dụ báo xoáy thuận nhiệt đới bằng mô hình số trị. Tạp chí Khoa học ĐHQGHN: Các Khoa học Trái đất và Môi trường, 32 (3S), 224-235.

6. Fujita, T., (1952), Pressure distribution within typhoon. The Geophys Geophysical Magazine, 23, 437-451.

7. Hiep, V.N., Chen, Y.L., (2011), High-Resolution Initialization and Simulations of Typhoon Morakot (2009). Monthly Weather Review, 139 (5), 1463-1491.

8. Hiep, V.N., Chen, Y.L., (2014), Improvements to a Tropical Cyclone Initialization Scheme and Impacts on Forecasts. Monthly Weather Review, 142 (11), 4340-4356. https://doi.org/10.1175/MWR-D-12-00329.1

9. Holland, G.J., (1980), An analytic model of the wind and pressure profiles in a hurricane. Monthly Weather Review. Monthly Weather Review, 108, 1212-1218.

10. Kurihara, Y., Bender, M.A., Ross, R.J., (1993), An initialization scheme of hurricane models by vortex specification. Monthly Weather Review, 121, 2030-2045.

11. Kurihara, Y., Bender, M.A., Tuleya, R.E., Ross, R.J., (1995), Improvements in the GFDL hurricane prediction system. Monthly Weather Review, 123, 2791-2801.

12. Kwon, I.H., Cheong, H.B., (2010), Tropical cyclone initialization with a spherical high-order filter and an idealized three-dimensional bogus vortex. Monthly Weather Review, 138, 1344-1367.

13. Mathur, M.B., (1991), The national meteorological center's quasiLagrangian model for hurricane prediction. Monthly Weather Review, 119, 1419-1447.

14. Nguyễn Thị Hoan, Hoàng Đức Cường, Trương Bá Kiên, Nguyễn Văn Hiệp, Kiều Quốc Chánh, Vijay Tallapragada, Nguyễn Tiến MạnhĐàng Hồng Như, Lã Thị Tuyết, Mai Văn Khiêm (2015), Vai trò của ban đầu hóa xoáy trong mô hình HWRF đối với mô phỏng cấu trúc bão Ketsana (2009). Tạp chí Khí tượng Thủy văn, 649, 7-11.

15. Phan Văn Tân, Nguyễn Lê Dũng (2008), Thử nghiệm ứng dụng hệ thống WRF-VAR kết hơpp với ban đầu hóa xoáy vào dụ báo quỹ đạo bão trên biển Đông. Tạp chí Khí tượng Thủy văn, 583, $1-9$.

16. Pu, Z.X., Tao, W.K., Braun, S., Simpson, J., Jia, Y., Halverson, J., Olson, W., Hou, A., (2002), The impact of TRMM data on mesoscale numerical simulation of Supertyphoon Paka. Monthly Weather Review, 130, 2448-2458.

17. Trần Tân Tiến, Lê Thị Hồng Vân (2009), Nghiên cưu ảnh hưởng của các yếu tố cấu thành xoáy nhân tạo trong đồng hóa số liệu xoáy giả bằng mô hình WRF đối với cơn bão Lêkima, Tạp chí Khoa học ĐHQGHN, Khoa học Tự nhiên và Công nghệ, 25 (33S), 508-516.

18. Wang, D., Liang, X., Zhao, Y., Wang, B., (2008), A comparison of two tropical cyclone bogussing schemes. Weather and Forecasting, 23, 194-204.

19. Wu, C.C., (2001), Numerical simulation of Typhoon Gladys (1994) and its interaction with Taiwan terrain using the GFDL hurricane model. Monthly Weather Review, 129, 1533-1549.

20. Xiao, Q., Zou, X., Wang, B., (2000), Initialization and simulation of a landfalling hurricane using a variational bogus data assimilation scheme. Monthly Weather Review, 128, 2252-2269.

21. Zhao, Y., Wang, B., Wang, Y., (2007), Initialization and simulation of a landfalling typhoon using a variational bogus mapped data assimilation (BMDA). Meteorology and Atmospheric Physics, 98(3-4), 269-282. 


\title{
BÀI BÁO KHOA HỌC
}

22. Zhang, S., Li, T., Ge, X., Peng, M., Pan, N., (2012), A 3DVAR-based dynamical initialization scheme for tropical cyclone predictions. Weather and Forecasting, 27, 473-483.

23. Zou, X., Xiao, Q., (2000), Studies on the initialization and simulation of a mature hurricane using a variational bogus data assimilation scheme. Journal of the Atmospheric Sciences, 57, 836860 .

\section{APPLICATION OF A DYNAMICAL VORTEX INITIALIZATION SCHEME ON SIMULATION AND STUDY OF STRUCTURE OF TY- PHOON MUJIGAE (2015) DURING NEAR-SHORE AND LANDFALL PERIOD \\ Nguyen Binh Phong ${ }^{1,2}$, Nguyen Tien Manh ${ }^{1}$, Nguyen Xuan Anh ${ }^{1}$ \\ Pham Le Khuong ${ }^{1}$, Nguyen Duc Nam ${ }^{1}$, Pham Xuan Thanh ${ }^{1}$, Nguyen Van Hiep ${ }^{1}$}

${ }^{1}$ Institute of Geophysics (IGP), Vietnam Academy of Science and Technology (VAST)

${ }^{2}$ Hanoi University of Natural Resources and Environment

\begin{abstract}
This study applies the dynamical vortex initialization (DVI) method with the Weather Research Forecasting (WRF) to investigate the effect of terrain and Northeast Monsoon on structure of typhoon Mujigae (2015) during the near-shore and landfall period. The results showed that the DVI significantly improves the quality of initial vortex with the size of eye and cloud structure agreed well with observation. Analysis of the model output with vortex initialzation shows that the vortex has a significantly asymmetrical structure. An abnormal feature in the structure of the Mujigae typhoon is that wind speeds over land where friction is large are stronger than over sea where friction is weak. This abnormal feature may be due to the fact that the winds in the northern part of the storm are strengthened by the strong gradient winds in the northern region of the typhoon when a continental high pressure center is presented.
\end{abstract}

Keywords: Dynamical Vortex Initialization (DVI), Typhoon, WRF model. 